VoL. 67 (2003) [305-315]

\title{
HARMONIC CHARACTERISTIC VECTOR FIELDS ON CONTACT METRIC THREE-MANIFOLDS
}

\author{
Domenico Perrone
}

\section{Dedicated to the memory of my father}

\begin{abstract}
In this paper we show that a contact metric three-manifold is a generalised $(k, \mu)$ space on an everywhere dense open subset if and only if its characteristic vector field $\xi$ determines a harmonic map from the manifold into its unit tangent sphere bundle equipped with the Sasaki metric. Moreover, we classify the contact metric threemanifolds whose characteristic vector field $\xi$ is strongly normal (or equivalently, is harmonic and minimal).
\end{abstract}

\section{INTRODUCTION}

Blair, Koufogiorgos and Papantoniou [2] introduced the so-called $(k, \mu)$-spaces. Such spaces are contact metric manifolds $(M, \eta, \xi, g, \phi)$ (for the definition of these manifolds, see Section 2) satisfying

$$
R(X, Y) \xi=k\{\eta(Y) X-\eta(X) Y\}+\mu\{\eta(Y) h X-\eta(X) h Y\}
$$

where $R$ is the curvature tensor, $k, \mu$ are constant and $2 h=L_{\xi} \phi$.

Recently, Koufogiorgos and Tsichlias [10] introduced a new class of contact metric three-manifolds: the generalised $(k, \mu)$-spaces. These spaces are defined by the above equation where $(k, \mu)$ are functions. They proved that, if $\operatorname{dim} M>3$, then $k$ and $\mu$ are necessarely constant and if $\operatorname{dim} M=3$, there exist examples of generalised $(k, \mu)$-spaces which are not $(k, \mu)$-spaces. However, they did not provide a classification for this new class and few results are known. One purpose of this paper is to give a characterisation of such spaces in terms of harmonic maps.

If $(M, g)$ is a Riemannian manifold and $\left(T^{1} M, g_{s}\right)$ is its unit tangent sphere bundle equipped with the Sasaki metric $g_{s}$, a unit vector field $V$ on $M$ determines a map between $(M, g)$ and $\left(T^{1} M, g_{s}\right)$. When $M$ is compact and orientable, the energy of $V$ is the energy $E(V)$ of the corresponding map. $V$ is said to be a harmonic vector field if it is a critical point for the energy functional $E$ defined on the space $\chi^{1}(M)$ of all unit vector fields

Received 16th November, 2002

Supported by funds of the University of Lecce and the M.U.R.S.T.

The author wishes to thank Lieven Vanhecke for his useful comments.

Copyright Clearance Centre, Inc. Serial-fee code: 0004-9727/03 \$A2.00+0.00. 
on $(M, g)$. The corresponding critical point condition has been determined in $[\mathbf{1 7}]$ and [18]. A harmonic vector field determines a harmonic map if an additional condition is satisfied ([9]). Gil-Medrano [6] introduced similar notions when $M$ is also non-compact and non-orientable. Now, it is well known that the characteristic vector field $\xi$ plays a fundamental role in the study of the Riemannian geometry of a contact metric manifold (see [1]). So, it is natural to study the harmonicity of $\xi$ in contact metric geometry. If $M$ is Sasakian, then $\xi$ is a harmonic vector field ([17]) and determines a harmonic map ([8]). Han and Yim [9] proved that the Hopf vector field $\xi$ on the unit 3-sphere $S^{3}$, that is the characteristic vector field of the standard contact metric structure on $S^{3}$ (see for example [1]), is the only unit vector field which defines a harmonic map into its unit tangent bundle. In [15], the present author proves that the characteristic vector field $\xi$ of a general contact metric manifold is a harmonic vector field if and only if it is an eigenvector of the Ricci operator. González-Dávila and Vanhecke [8] studied harmonicity and minimality of the characteristic vector field of a contact metric threemanifold when the space is locally homogeneous or has constant scalar curvature. The same authors introduced in [7] the notion of a strongly normal unit vector field $V$. Such notion generalises the normality notion used in contact metric geometry where the role of $V$ is played by the characteristic vector field.

The main results of this paper are the following.

ThEOREM 1.1. Let $(M, g, \eta, \xi, \phi)$ be a contact metric three-manifold. Then the characteristic vector field $\xi:(M, g) \rightarrow\left(T^{1} M, g_{s}\right)$ defines a harmonic map if and only if $M$ is a generalised $(k, \mu)$-space on an everywhere dense open subset (specified in Section 2).

For a non-Sasakian contact metric three-manifold, we define the invariant $p$ $:=(4 \sqrt{2} W) /\|\tau\|$, where $W$ is the Webster scalar curvature and $\|\tau\|$ the scalar torsion introduced by Chern and Hamilton [4]. Then, we get the following.

TheOREM 1.2. Let $(M, \eta, g, \xi, \phi)$ be a contact metric three-manifold. Then the following statements are equivalent:

(i) $\xi$ is strongly normal;

(ii) $\xi$ is harmonic and minimal;

(iii) $M$ is Sasakian or is locally isometric to a unimodular Lie group $G$ equipped with a non-Sasakian left-invariant contact metric structure $(\eta, g)$. More precisely:

if $p>1, \widetilde{G}$ is the 3-sphere group $S U(2)$;

if $p=1, \widetilde{G}$ is the group $\widetilde{E}(2)$, that is, the universal covering of the group of rigid motions of Euclidean 2-space;

if $-1 \neq p<1, \widetilde{G}$ is the group $\widetilde{S L}(2, R)$;

if $p=-1, \widetilde{G}$ is the group $E(1,1)$ of rigid motions of the Minkowski 2-space; 
where $\widetilde{G}$ denotes the universal covering of $G$.

COROLlaRY 1.3. A compact three-manifold admits a contact metric structure whose characteristic vector field is strongly normal, or equivalently, harmonic and minimal, if and only if it is diffeomorphic to a left quotient of the Lie group $G$ under a discrete subgroup, where $G$ is one of $S U(2), H^{3}$ (the Heisenberg group), $\widetilde{S L}(2, R), \widetilde{E}(2)$ or $E(1,1)$.

\section{Preliminaries on CONTACT Metric ManifoldS}

In this section, we collect some basic facts about contact metric manifolds. All manifolds are assumed to be connected and smooth. A $(2 n+1)$-dimensional manifold $M$ is said to be a contact manifold if it admits a global 1-form $\eta$ such that $\eta \wedge(d \eta)^{n} \neq 0$. Given $\eta$, there exists a unique vector field $\xi$, called the characteristic vector field or the Reeb vector field, such that $\eta(\xi)=1$ and $d \eta(\xi, \cdot)=0$. Furthermore, a Riemannian metric $g$ is said to be an associated metric if there exists a tensor $\phi$ of type $(1,1)$ such that

$$
\eta=g(\xi, \cdot), d \eta(\cdot, \cdot)=g(\cdot, \phi \cdot), \phi^{2}=-I^{2}+\eta \otimes \xi .
$$

$(\eta, \xi, \phi)$ is called an almost contact structure. $(\eta, g, \xi, \phi)$, or $(\eta, g)$, is called a contact metric structure and $(M, \eta, g, \xi, \phi)$ a contact metric manifold. We denote by $\nabla$ the Levi Civita connection and by $R$ the corresponding Riemann curvature tensor given by

$$
R_{X Y}=\nabla_{[X, Y]}-\left[\nabla_{X}, \nabla_{Y}\right]
$$

for all smooth vector fields $X, Y$. Moreover, we denote by $\rho$ the Ricci tensor of type $(0,2)$, by $Q$ the corresponding endomorphism field and by $r$ the scalar curvature. The tensor $h=\left(L_{\xi} \phi\right) / 2$, where $L$ denotes the Lie derivative, is symmetric and satisfies

$$
\nabla \xi=-\phi-\phi h, \quad \nabla_{\xi} \phi=0, \quad h \phi=-\phi h, \quad h \xi=0 .
$$

The tensor $h$ and the scalar torsion $\|\tau\|, \tau=L_{\xi} g$, introduced in [4], are related by

$$
\tau=2 g(h \phi, \cdot), \quad\|\tau\|^{2}=4 \operatorname{tr} h^{2}
$$

If $M$ is 3-dimensional, and $0, \lambda,-\lambda$ are the eigenvalues of $h$, then $\|\tau\|^{2}=8 \lambda^{2}=8-4 \rho(\xi, \xi)$.

A contact metric manifold is said to be a $K$-contact manifold if $\xi$ is a Killing vector field, or equivalently, $h=0$. An almost contact structure $(\eta, \xi, \phi)$ naturally gives an almost complex structure on the product manifold $M \times R$. If this almost complex structure is integrable, the almost contact structure is called normal. If an almost contact structure comes from a contact form $\eta$, then the contact form $\eta$ is called normal. A Sasakian manifold is a normal contact metric manifold. Moreover, a contact metric structure $(\xi, \eta, \phi, g)$ is a Sasakian structure if and only if

$$
\left(\nabla_{X} \phi\right) Y=g(X, Y) \xi-\eta(Y) X
$$


Any Sasakian manifold is $K$-contact and the converse also holds when $n=1$, that is, for three-dimensional spaces. We refer to [1] for more information about contact metric manifolds.

Next, let $(M, \eta, g, \xi, \phi)$ be a contact metric three-manifold and $m$ a point of $M$. Then there exists a smooth local orthonormal basis of the form $\left\{\xi, e_{1}, e_{2}=\phi e_{1}\right\}$ in a neigborhood of $m$. Now, let $U_{1}$ be the open subset of $M$ where $h \neq 0$ and let $U_{2}$ be the open subset of points $m \in M$ such that $h=0$ in a neighbourhood of $m . U_{1} \cup U_{2}$ is an open dense subset of $M$. On $U_{1}$ we put $h e_{1}=\lambda e_{1}$ and hence we have $h e_{2}=-\lambda e_{2}$ where $\lambda$ is a non-vanishing smooth function. Then, we have

LEMMA 2.1. [3] On $U_{1}$ we have

$$
\begin{array}{ll}
\nabla_{\xi} e_{1}=-a e_{2}, & \nabla_{\xi} e_{2}=a e_{1}, \\
\nabla_{e_{1}} \xi=-(\lambda+1) e_{2}, & \nabla_{e_{2}} \xi=-(\lambda-1) e_{1}, \\
\nabla_{e_{1}} e_{1}=\frac{1}{2 \lambda}\left\{e_{2}(\lambda)+A\right\} e_{2}, & \nabla_{e_{2}} e_{2}=\frac{1}{2 \lambda}\left\{e_{1}(\lambda)+B\right\} e_{1}, \\
\nabla_{e_{1}} e_{2}=-\frac{1}{2 \lambda}\left\{e_{2}(\lambda)+A\right\} e_{1}+(\lambda+1) \xi, \\
\nabla_{e_{2}} e_{1}=-\frac{1}{2 \lambda}\left\{e_{1}(\lambda)+B\right\} e_{2}+(\lambda-1) \xi \\
\left.\left[e_{1}, e_{2}\right]=-\frac{1}{2 \lambda}\left\{e_{2}(\lambda)+A\right)\right\} e_{1}+\frac{1}{2 \lambda}\left\{e_{1}(\lambda)+B\right\} e_{2}+2 \xi,
\end{array}
$$

where $A=\rho\left(\xi, e_{1}\right), B=\rho\left(\xi, e_{2}\right)$ and $a$ is a smooth function. Moreover,

$$
\nabla_{\xi} h=2 a h \phi+\xi(\lambda) s,
$$

where $s$ is the tensor field defined by $s \xi=0, s e_{1}=e_{1}, s e_{2}=-e_{2}$

Finally, we recall that the components of the Ricci operator $Q$, with respect to $\left\{\xi, e_{1}, e_{2}=\phi e\right\}$, are given by (see [13])

$$
\left\{\begin{array}{l}
Q \xi=2\left(1-\lambda^{2}\right) \xi+A e_{1}+B e_{2}, \\
Q e_{1}=A \xi+\left(\frac{r}{2}-1+\lambda^{2}+2 a \lambda\right) e_{1}+\xi(\lambda) e_{2} \\
Q e_{2}=B \xi+\xi(\lambda) e_{1}+\left(\frac{r}{2}-1+\lambda^{2}-2 a \lambda\right) e_{2} .
\end{array}\right.
$$

3. GENERALISED $(k, \mu)$-SPACES AND HARMONICITY OF THE CHARACTERISTIC VECTOR FIELD

Let $(M, g, \eta, \xi, \phi)$ be a contact metric three-manifold. We recall that $M$ is called generalised $(k, \mu)$-space if

$$
R(X, Y) \xi=k\{\eta(X) Y-\eta(Y) X\}+\mu\{\eta(X) h Y-\eta(Y) h X\},
$$


where $k$ and $\mu$ are in general smooth functions. If $k=1$, then $M$ is Sasakian. If $k$ and $\mu$ are constant, $M$ is a $(k, \mu)$-space.

Moreover, we recall that a unit vector field $V$ on a Riemannian manifold $(M, g)$ defines a harmonic map $V:(M, g) \rightarrow\left(T^{1} M, g_{s}\right)$ if and only if the following two conditions are satisfied:

(1) $V$ is a harmonic vector field, and

(2) $\operatorname{trR}(\nabla \cdot V, V) \cdot=0$.

(See [9] for the compact case and [6] for the non-compact case.)

In [15] contact metric manifolds whose characteristic vector field $\xi$ is a harmonic vector field are called $H$-manifolds. Moreover, we have

THEOREM 3.1. [15] The characteristic vector field of a contact metric $(2 n+1)$ manifold is a harmonic vector field if and ony if it is an eigenvector of the Ricci operator. For $n=1$, this theorem is proved in [8].

For a contact metric three-manifold, from Lemma 2.1, we have on $U_{1}$ :

$$
\operatorname{trR}(\nabla . \xi, \xi) \cdot=-2 \lambda \xi(\lambda) \xi+(\lambda-1) B e_{1}+(\lambda+1) A e_{2},
$$

and hence, by Theorem 3.1, we obtain easily the following

Proposition 3.2. [8] Let $(M, g, \eta, \xi, \phi)$ be a contact metric three-manifold. Then $\xi$ defines a harmonic map if and only if $\xi$ is a harmonic vector field and $\xi\|\tau\|^{2}=0$.

In order to prove Theorem 1.1, we first prepare some lemmas.

Lemma 3.3. Let $(M, g, \eta, \xi, \phi)$ be a generalised $(k, \mu)$-space. Then on $U_{1} \cup U_{2}$ we have

$$
\nabla_{\xi} h=\mu h \phi, \quad \mu=2 a, \quad \xi(\lambda)=0, \quad k=1-\lambda^{2},
$$

where $a$ and $\lambda$ are the functions defined in Lemma 2.1.

Proof: We use the notations of Lemma 2.1. If the open set $U_{2}$ is not empty, then the restriction of the contact structure to $U_{2}$ is Sasakian and in this case $h=0, \lambda=a=\mu=0$ and $k=1$. Furthermore, on $U_{1}$ the equation (3.1) implies that the operator $\ell:=R(\xi, \cdot) \xi$ satisfies

$$
\ell=-k \phi^{2}+\mu h=k(I-\eta \otimes \xi)+\mu h
$$

This equation implies

$$
\ell \phi=k \phi+\mu h \phi \quad \text { and } \quad \phi \ell=k \phi-\mu h \phi,
$$

from which follows

$$
\ell \phi+\phi \ell=2 k \phi
$$


and

$$
\ell \phi-\phi \ell=2 \mu h \phi .
$$

Moreover, for a general contact metric manifold, we have (see [12, formula (2.6)])

$$
2 \nabla_{\xi} h=\ell \phi-\phi \ell .
$$

Hence, (3.4) gives

$$
\nabla_{\xi} h=\mu h \phi \quad \text { or equivalently, } \quad \phi \nabla_{\xi} h=\mu h .
$$

So, (2.4) of Lemma 2.1 and (3.5) give $\mu=2 a$ and $\xi(\lambda)=0$. Moreover, from (3.2) and (3.5), we get

$$
\ell=-k \phi^{2}+\phi \nabla_{\xi} h
$$

which is equivalent (see $[\mathbf{1 2}$, Remark 2.4]) to

$$
h^{2}=(k-1) \phi^{2} .
$$

This gives $k=1-\lambda^{2}$.

LEMmA 3.4. If $M$ is a generalised $(k, \mu)$-space, then $\rho(\xi, \cdot)_{\mid \operatorname{ker} \eta}=0$, that is, $\xi$ is a harmonic vector field.

Proof: From (3.1) we have $R(X, Y) \xi=0$ for all $X, Y \in \operatorname{ker} \eta$ and hence $\rho(X, \xi)$ $=0$ for all $X \in \operatorname{ker} \eta$.

Proof of the Theorem 1.1: We use the notations of Lemma 2.1. If the open set $U_{2}$ is not empty, then the restriction of the contact structure to $U_{2}$ is Sasakian and in this case the theorem is trivial. Next, let $U_{1}$ be non-empty and let $\left(\xi, e_{1}, e_{2}=\phi e_{1}\right)$ be a local $\phi$-basis as in Lemma 2.1 .

Assume that $\mathrm{M}$ is a generalised $(k, \mu)$-space. Then from Proposition 3.2 and Lemmas 3.4 and 3.5, it follows that $\xi$ defines a harmonic map. Conversely, assume that $\xi$ defines a harmonic map. We show that $\mathrm{M}$ is a generalised $(k, \mu)$-space on $U_{1}$. In [13], it is proved that the Ricci operator $Q$ of a general contact metric three-manifold is (locally) given by

$$
Q=\alpha I+\beta \eta \otimes \xi+\phi \nabla_{\xi} h-\sigma\left(\phi^{2}\right) \otimes \xi+\sigma\left(e_{1}\right) \eta \otimes e_{1}+\sigma\left(e_{2}\right) \eta \otimes e_{2}
$$

where $\sigma=\rho(\xi, \cdot)_{\mid \text {ker } \eta,}, \alpha=(r / 2)-1+\lambda^{2}$ and $\beta=-(r / 2)+3-3 \lambda^{2}$. Since (2.4)holds, from (3.7) we obtain on $U_{1}$ that $\sigma=0$ and $\xi(\lambda)=0$ if and only if

$$
Q=\left\{\frac{r}{2}-1+\lambda^{2}\right\} I+\left\{-\frac{r}{2}+3-3 \lambda^{2}\right\} \eta \otimes \xi+2 a h .
$$

Moreover, for a Riemannian three-manifold the curvature $R(X, Y) \xi$ is completely determined by the Ricci operator. More precisely, we have

$$
R(X, Y) \xi=\eta(X) Q Y-\eta(Y) Q X-g(Q Y, \xi) X+g(Q X, \xi) Y-\frac{r}{2}\{\eta(X) Y-\eta(Y) X\}
$$


and hence, using (3.8), we get

$$
R(X, Y) \xi=\left(1-\lambda^{2}\right)\{\eta(X) Y-\eta(Y) X\}+2 a\{\eta(X) h Y-\eta(Y) h X\} .
$$

Therefore, $M$ is a generalised $(k, \mu)$-space with $k=1-\lambda^{2}$ and $\mu=2 a$ on $U_{1}$.

REMARK. By the proof of Theorem 1.1, we get that, for a contact metric three-manifold, the following properties are equivalent on $U_{1} \cup U_{2}$ :

(1) $M^{3}$ is a generalised $(k, \mu)$-space;

(2) $\rho(\xi, \cdot)_{\mid \operatorname{ker} \eta}=0$ and $\xi(\lambda)=0$;

(3) $Q=((r / 2)-k) I+(-(r / 2)+3 k) \eta \otimes \xi+\mu h$.

EXAmples. Consider the three-dimensional manifold $M=\left\{x \in R^{3}: x_{3} \neq 0\right\}$ and the vector fields

$$
e_{1}=\frac{\partial}{\partial x_{1}}, \quad e_{2}=\frac{1}{x_{3}^{2}} \frac{\partial}{\partial x_{2}}, e_{3}=2 x_{2} x_{3}{ }^{2} \frac{\partial}{\partial x_{1}}+\frac{2 x_{1}}{x_{3}^{6}} \frac{\partial}{\partial x_{2}}+\frac{1}{x_{3}^{6}} \frac{\partial}{\partial x_{3}} .
$$

We define $\eta, \xi, g, \phi$ by $\xi=e_{1}, g\left(e_{i}, e_{j}\right)=\delta_{i j}, \eta(X)=g(\xi, X)$ and $\phi\left(e_{1}\right)=0, \phi\left(e_{2}\right)$ $=e_{3}, \phi\left(e_{3}\right)=-e_{2}$. Then $(M, \eta, \xi, g, \phi)$ is a generalised $(k, \mu)$-space (see [10]) and thus $\xi:(M, g) \longrightarrow\left(T^{1} M, g_{s}\right)$ defines a harmonic map. On the other hand, $\rho(\xi, \cdot)_{\mid k e r \eta}$ $=0$ and $\xi(\lambda)=0$ are both invariant under a $D$-homothetic deformation: $\eta_{t}=t \eta, \xi_{t}$ $=(1 / t) \xi, g_{t}=t g+t(t-1) \eta \otimes \eta, \phi_{t}=\phi, t>0$, and hence, for each positive number, there exists a contact metric three-manifold whose characteristic vector field defines a harmonic map.

4. HARMONIC, MINIMAL AND STRONGLY NORMAL CHARACTERISTIC VECTOR FIELDS ON CONTACT METRIC THREE-MANIFOLDS

In [7], the authors introduced the notion of a strongly normal unit vector field. A unit vector field $V$ on a Riemannian manifold is called strongly normal if

$$
g\left(\left(\nabla_{X}(\nabla V) Y, Z\right)=0 \text { for all } X, Y, Z \perp V .\right.
$$

Most of the results obtained in [7] are based on this notion because a strongly normal unit vector field is minimal. A unit vector field $V$ on a Riemannian manifold $(M, g)$ determines a submanifold of its unit tangent sphere bundle. When $M$ is compact and orientable, the volume of $V$ is the volume of the submanifold and $V$ is called minimal if it is critical for the volume functional defined on the space $\chi^{1}(M)$ of all unit vector fields on $(M, g)$. A similar notion has been introduced in [6] when $M$ is also non-compact and non-orientable.

The notion of a strongly normal unit vector field generalises the normality notion used in contact metric geometry where the role of $V$ is played by the characteristic vector 
field $\xi$. In fact, a normal contact metric manifold satisfies the conditon $\nabla \xi=-\phi$ and thus we have

$$
\nabla_{X}(\nabla \xi) Y=-\left(\nabla_{X} \phi\right) Y=\eta(Y) X-g(X, Y) \xi
$$

In [8], the same authors study harmonicity and minimality of the characteristic vector field of a contact metric three-manifold $(M, \eta, g, \xi, \phi)$. They proved the following results. If $M$ is locally homogeneous, then $\xi$ is harmonic if and only if it is minimal or equivalently, if and only if $M$ is locally isometric to a unimodular Lie group equipped with a left-invariant contact metric structure. If $M$ has constant scalar curvature, then $M$ is locally isometric to a unimodular Lie group equipped with a left-invariant contact metric structure if and only if $\xi$ is harmonic and minimal. Theorem 1.2, Corollary 1.3 and Proposition 4.1 extend such results.

ProOF OF THE THEOREM 1.2: Let $(M, \eta, g, \xi, \phi)$ be a contact metric threemanifold. We use the notations of Lemma 2.1. If the open set $U_{2}$ is not empty, then the restriction of the contact structure to $U_{2}$ is Sasakian and in this case $\xi$ is strongly normal, harmonic and minimal. Next, let $U_{1}$ be non-empty and let $\left(\xi, e_{1}, e_{2}\right)$ be a local $\phi$-basis on $U_{1}$ as in Lemma 2.1. From (2.1) it follows that $-\nabla_{X} \nabla \xi$ $=\nabla_{X} \phi+\nabla_{X}(\phi h)=\nabla_{X} \phi+\left(\nabla_{X} \phi\right) h+\phi\left(\nabla_{X} h\right)$. In dimension 3 , the $C R$-structure associated to the contact metric structure is integrable (see for example [1]), that is $\left(\nabla_{X} \phi\right) Y=g(X+h X, Y) \xi-\eta(X)(X+h X)$. Therefore,

$\xi$ is strongly normal $\Longleftrightarrow\left(\nabla_{X} h\right) Y$ is collinear to $\xi$ for all $X, Y \in \operatorname{ker} \eta$.

From Lemma 2.1, we have

$$
\begin{aligned}
& \left(\nabla_{e_{1}} h\right) e_{1}=\nabla_{e_{1}} h e_{1}-h\left(\nabla_{e_{1}} h e_{1}\right)=e_{1}(\lambda) e_{1}+\left(e_{2}(\lambda)+A\right) e_{2} \\
& \left(\nabla_{e_{1}} h\right) e_{2}=\nabla_{e_{1}} h e_{2}-h\left(\nabla_{e_{1}} h e_{2}\right)=-\lambda(\lambda+1) \xi+\frac{1}{2}\left(e_{2}(\lambda)+B\right) e_{1}-e_{1}(\lambda) e_{2} \\
& \left(\nabla_{e_{2}} h\right) e_{2}=\nabla_{e_{2}} h e_{2}-h\left(\nabla_{e_{2}} h e_{2}\right)=-\left(e_{2}(\lambda)+B\right) e_{1}-e_{2}(\lambda) e_{2} \\
& \left(\nabla_{e_{2}} h\right) e_{1}=\nabla_{e_{2}} h e_{1}-h\left(\nabla_{e_{2}} h e_{1}\right)=e_{2}(\lambda) e_{1}-\left(e_{1}(\lambda)+B\right) e_{2}
\end{aligned}
$$

So,

$$
\xi \text { is strongly normal on } U_{1} \Longleftrightarrow e_{1}(\lambda)=e_{2}(\lambda)=A=B=0 \text {. }
$$

Now, in [8], the authors derived the following analityc condition for the minimality of $\xi$. The characteristic vector field $\xi$ is minimal if and only if on the open $U_{1}$ we have

$$
\left\{\begin{array}{l}
A=4\left\{\lambda\left(\lambda^{2}+2(1-\lambda)\right)\right\}^{-1} e_{2}(\lambda) \\
B=4\left\{\lambda\left(\lambda^{2}+2(1+\lambda)\right)\right\}^{-1} e_{1}(\lambda)
\end{array}\right.
$$


Consequently, on $U_{1}$ we have

$\xi$ is strongly normal $\Longleftrightarrow \xi$ is harmonic and minimal.

Moreover, by $(2.3),(4.1)$ implies $2 \xi(\lambda)=\left[e_{1}, e_{2}\right](\lambda)=0$. Thus, we get on $U_{1}$ :

$\xi$ is strongly normal $\Longleftrightarrow \lambda$ is constant and $\xi$ is harmonic.

In this case, that is when $\xi$ is strongly normal, (2.5) becomes

$$
\left\{\begin{array}{l}
Q \xi=2\left(1-\lambda^{2}\right) \xi \\
Q e_{1}=\left(\frac{r}{2}-1+\lambda^{2}+2 a \lambda\right) e_{1} \\
Q e_{2}=\left(\frac{r}{2}-1+\lambda^{2}-2 a \lambda\right) e_{2}
\end{array}\right.
$$

from which we easily get

$$
\left\{\begin{array}{l}
\left(\nabla_{\xi} Q\right) \xi=0 \\
\left(\nabla_{e_{1}} Q\right) e_{1}=\left(e_{1}\left(\frac{r}{2}\right)+2 \lambda e_{1}(a)\right) e_{1} \\
\left(\nabla_{e_{2}} Q\right) e_{2}=\left(e_{2}\left(\frac{r}{2}\right)+2 \lambda e_{2}(a)\right) e_{2} .
\end{array}\right.
$$

Then, using the formula

$$
\frac{1}{2} X(r)=\sum_{i} g\left(\left(\nabla_{E_{i}} Q\right) E_{i}, X\right)
$$

where $\left\{E_{i}\right\}$ is an orthonormal basis, we get

$$
\left\{\begin{array}{l}
e_{1}\left(\frac{r}{2}\right)=e_{1}\left(\frac{r}{2}\right)+2 \lambda e_{1}(a) \\
e_{2}\left(\frac{r}{2}\right)=e_{2}\left(\frac{r}{2}\right)+2 \lambda e_{2}(a)
\end{array}\right.
$$

So, $e_{1}(a)=e_{2}(a)=0$ and hence, since $2 \xi=\left[e_{1}, e_{2}\right], a$ is locally constant on $U_{1}$. Since $\lambda$ is continuous, it follows that $M=U_{1}$ and hence $\lambda$ and $a$ are globally constant. Then, Lemma 2.1 gives

$$
\left[\xi, e_{1}\right]=c_{2} e_{2},\left[\xi, e_{2}\right]=c_{1} e_{1} \text { and }\left[e_{1}, e_{2}\right]=2 \xi \text {, }
$$

where $c_{1}=\lambda+a-1$ and $c_{2}=\lambda-a+1$ are constant. From this we obtain that $M$ is locally isometric to a unimodular Lie group with a left-invariant contact metric structure (see [16, p. 10], [11] and [14, Theorem 3.1]). [14, Theorem 3.1] gives a classification of the unimodular Lie groups with a left-invariant contact metric structure in terms of the Webster scalar curvature $W=(r-\operatorname{Ric}(\xi, \xi)+4) / 8=\left(r+2+\|\tau\|^{2} / 4\right) / 8$ and the scalar torsion $\|\tau\|$. Since our contact metric structure is non-Sasakian, then we can consider the invariant $p:=(4 \sqrt{2} W) /\|\tau\|$ and (1) of [14, Theorem 3.1] can be reformulated as (iii) of Theorem 1.2 . 
ProOF OF Corollary 1.3. Geiges [5] proved that a compact three-manifold admits a normal contact form (that is, a Sasakian structure, see Section 2) if and only if it is diffeomorphic to a left quotient $\Gamma \backslash G$ of a Lie group $G$ under a discrete subgroup $\Gamma$, where $\mathrm{G}$ is one of $S U(2), H^{3}$ (the Heisenberg group), $\widetilde{S L}(2, R)$. This result and Theorem 1.2 imply the Corollary 1.3 .

REMARK. Let $G$ be one of the following simply connected unimodular Lie groups: $S U(2)$, $H^{3}, \widetilde{S L}(2, R), \widetilde{E}(2)$ or $E(1,1)$. Consequently, $G$ contains a discrete subgroup $\Gamma$ such that the space of right cosets $\Gamma \backslash G$ is a differentiable manifold and the natural projection $\pi$ is a differentiable map. Moreover, each left-invariant vector field on $G$ descends to $\Gamma \backslash G$, or equivalently, if $X$ is left-invariant, then $\pi_{\star} X_{b a}=\pi_{\star} X_{a}$, for all $a \in G$ and $b \in \Gamma$. In a similar way, a left invariant contact metric structure on $G$ and, in general, all its left-invariant tensor fields, descend to the quotient space. So, if we consider on $G$ a leftinvariant contact metric structure (see [14]), then $\Gamma \backslash G$ has a contact metric structure with the same curvature properties for the curvature tensor on $G$. Also, the projections of the (left-invariant) characteristic vector fields preserve the properties of being harmonic, minimal and to determine harmonic maps into the corresponding unit tangent sphere bundle. Note that a three-dimensional Lie group $G$ admits a discrete subgroup $\Gamma$ such that $\Gamma \backslash G$ is compact if and only if $G$ is unimodular ([11]).

We conclude this section with the following.

Proposition 4.1. Let $M$ be a contact metric three-manifold with constant scalar torsion $\|\tau\|$. Then $\xi$ is harmonic $\Longleftrightarrow \xi$ is minimal $\Longleftrightarrow \xi$ is strongly normal $\Longleftrightarrow M$ is Sasakian or it is strongly locally $\phi$-symmetric.

Proof: We use the notations of Lemma 2.1. The conditon $\|\tau\|=$ constant is equivalent to the condition $\lambda=$ constant. So, from (4.1), (4.2) and Theorem 3.1 follows that harmonicity, minimality and strongly normality for $\xi$ are equivalent. Finally, from [3, Section 5] it follows that the three-dimensional strongly locally $\phi$-symmetric spaces are locally isometric to the Lie groups listed in Theorem 1.2.

\section{REFERENCES}

[1] D.E. Blair, Riemannian geometry of contact and symplectic manifold, Progress in Math. 203 (Birkhäuser, Boston, Basel, Berlin, 2002).

[2] D.E. Blair, T. Koufogiorgos and B.J. Papantoniou, 'Contact metric manifolds satisfying a nullity condition', Israel J. Math. 91 (1995), 189-214.

[3] G. Calvaruso, D. Perrone and L. Vanhecke, 'Homogeneity on three-dimensional contact metric manifolds', Israel J. Math. 114 (1999), 301-321.

[4] S.S. Chern and R.S. Hamilton, On Riemannian metrics adapted to three-dimensional contact manifolds, Lecture Notes in Math. 1111 (Springer-Verlag, Berlin, Heidelberg, New York, 1985).

[5] H. Geiges, 'Normal contact structure on 3-manifolds', Tôhoku Math. J. 49 (1997), 415-422. 
[6] O. Gil-Medrano, 'Relationiship between volume and energy of unit vector fields', Differential Geom. Appl. 15 (2001), 137-152.

[7] J.C. González-Dávila and L. Vanhecke, 'Examples of minimal unit vector fields', Ann. Global Anal. Geom. 18 (2000), 385-404.

[8] J.C. González-Dávila and L. Vanhecke, 'Minimal and harmonic characteristic vector fields on three-dimensional contact metric manifolds', J. Geom. 72 (2001), 65-76.

[9] S.D. Han and J.W. Yim, 'Unit vector fields on spheres which are harmonic maps', Math. Z. 227 (1998), 83-92.

[10] T. Koufogiorgos and C. Tsichlias, 'On the existence of a new class of contact metric manifolds', Canad. Math. Bull. 43 (2000), 440-447.

[11] J. Milnor, 'Curvature of left invariant metrics on Lie groups', Adv. Math. 21 (1976), 293-329.

[12] D. Perrone, 'Contact Riemannian manifolds satisfying $R(X, \xi) \mathrm{R}=0$ ', Yokohama Math. $J$. 39 (1992), 141-149.

[13] D. Perrone, 'Ricci tensor and spectral rigidity of contact Riemannian three-manifolds', Bull. Inst. Math. Acad. Sinica 24 (1996), 127-138.

[14] D. Perrone, 'Homogeneous contact Riemannian three-manifolds', Ilinois Math. J. 42 (1998), 243-256.

[15] D. Perrone, 'Contact metric manifolds whose characteristic vector field is a harmonic vector field', (in preparation).

[16] F. Tricerri and L. Vanhecke, Homogeneous structures on Riemannian manifolds, London Math. Soc. Lect. Note Series 83 (Cambridge Univ. Press, Cambridge, 1983).

[17] G. Wiegmink, 'Total bending of vector fields on Riemannian manifolds', Math. Ann. 303 (1995), 325-344.

[18] C.M. Wood, 'On the energy of a unit vector field', Geom. Dedicata 64 (1997), 319-330.

Dipartimento di Matematica

Universitá degli Studi di Lecce

Via Provinciale Lecce-Arnesano

73100 Lecce

Italy

e-mail: domenico.perrone@unile.it 